\title{
Increasing the field-of-view of row-column-addressed ultrasound transducers: implementation of a diverging compound lens
}

Engholm, Mathias; Beers, Christopher; Bouzari, Hamed; Jensen, Jørgen Arendt; Thomsen, Erik Vilain

Published in:

Ultrasonics

Link to article, DOI:

10.1016/j.ultras.2018.02.001

Publication date:

2018

Document Version

Peer reviewed version

Link back to DTU Orbit

\section{Citation (APA):}

Engholm, M., Beers, C., Bouzari, H., Jensen, J. A., \& Thomsen, E. V. (2018). Increasing the field-of-view of row-column-addressed ultrasound transducers: implementation of a diverging compound lens. Ultrasonics, 88 , 97-105. https://doi.org/10.1016/j.ultras.2018.02.001

\section{General rights}

Copyright and moral rights for the publications made accessible in the public portal are retained by the authors and/or other copyright owners and it is a condition of accessing publications that users recognise and abide by the legal requirements associated with these rights.

- Users may download and print one copy of any publication from the public portal for the purpose of private study or research.

- You may not further distribute the material or use it for any profit-making activity or commercial gain

- You may freely distribute the URL identifying the publication in the public portal 


\title{
Increasing the Field-of-View of Row-Column-Addressed Ultrasound Transducers: Implementation of a Diverging Compound Lens
}

\author{
Mathias Engholma ${ }^{\mathrm{a}}$, Christopher Beers ${ }^{\mathrm{b}}$, Hamed Bouzaric $^{\mathrm{c}}$, Jørgen Arendt Jensen ${ }^{\mathrm{c}}$, Erik Vilain Thomsen ${ }^{\mathrm{a}}$ \\ ${ }^{a}$ Department of Micro- and Nanotechnology, DTU Nanotech, Technical University of Denmark, Building 345C, DK-2800 Kgs. Lyngby, Denmark \\ ${ }^{b}$ Sound Technology Inc., Analogic Ultrasound Group, State College, PA 16803, USA \\ ${ }^{c}$ Center for Fast Ultrasound Imaging, Department of Electrical Engineering, Technical University of Denmark, Building 349, DK-2800 Kgs. Lyngby, Denmark
}

\begin{abstract}
The purpose of this work is to investigate compound lenses for row-column-addressed (RCA) ultrasound transducers for increasing the field-of-view (FOV) to a curvilinear volume region, while retaining a flat sole to avoid trapping air between the transducer sole and the patient, which would otherwise lead to unwanted reflections. The primary motivation behind this research is to develop a RCA ultrasound transducer for abdominal or cardiac imaging, where a curvilinear volume region is a necessity. RCA transducers provide 3-D ultrasound imaging with fewer channels than fully-addressed 2-D arrays ( $2 N$ instead of $N^{2}$ ), but they have inherently limited FOV. By increasing the RCA FOV, these transducers can be used for the same applications as fully-addressed transducers while retaining the same price range as conventional 2-D imaging due to the lower channel count. Analytical and finite element method (FEM) models were employed to evaluate design options. Composite materials were developed by loading polymers with inorganic powders to satisfy the corresponding speed of sound and specific acoustical impedance requirements. $\mathrm{A} \mathrm{Bi}_{2} \mathrm{O}_{3}$ powder with a density of $8.9 \mathrm{~g} / \mathrm{cm}^{3}$ was used to decrease the speed of sound of a room temperature vulcanizing (RTV) silicone, RTV615, from $1.03 \mathrm{~mm} / \mu \mathrm{s}$ to $0.792 \mathrm{~mm} / \mu \mathrm{s}$. Using micro-balloons in RTV615 and a urethane, Hapflex 541, their speeds of sound were increased from $1.03 \mathrm{~mm} / \mu \mathrm{s}$ to $1.50 \mathrm{~mm} / \mu$ s and from $1.52 \mathrm{~mm} / \mu \mathrm{s}$ to $1.93 \mathrm{~mm} / \mu \mathrm{s}$, respectively. A diverging add-on lens was fabricated of a $\mathrm{Bi}_{2} \mathrm{O}_{3}$ loaded RTV615 and an unloaded Hapflex 541. The lens was tested using a RCA probe, and a FOV of $32.2^{\circ}$ was measured from water tank tests, while the FEM model yielded $33.4^{\circ}$. A wire phantom with $0.15 \mathrm{~mm}$ diameter wires was imaged at $3 \mathrm{MHz}$ down to a depth of $14 \mathrm{~cm}$ using a synthetic aperture imaging sequence with single element transmissions. The beamformed image showed that wires outside the array footprint were visible, demonstrating the increased FOV.
\end{abstract}

Keywords: Ultrasound Imaging, Compound Lens, Composite Materials, Diverging Lens, Row-column-addressing

\section{Introduction}

The purpose of this work is to investigate the use of compound lenses for row-column-addressed (RCA) ultrasound transducers for increasing the field-of-view (FOV) to a curvilinear volume region, while retaining a flat sole to avoid trapping air between the transducer sole and the patient, which leads to unwanted reflections. Planar 2-D RCA arrays can be an attractive alternative to fully-addressed matrix arrays, as they offer volumetric imaging with $2 N$ electrical connections compared to $N^{2}$ for fully-addressed matrix arrays. E.g. a $128 \times 128$ matrix array requires 16,384 connections, if every element needs to be connected, while by row-column-addressing only 256 connections are required. Seo and Yen presented simulation and experimental results of a $5 \mathrm{MHz}$ flat RCA array with $256+256$ element [1]. They imaged rectilinear volumes of nylon wire pairs and an $8 \mathrm{~mm}$ diameter cylindrical anechoic cyst phantom to verify the feasibility of the row-column-addressing scheme of matrix arrays. They showed that the wire spread function had a full width at half maximum of $0.68 \mathrm{~mm}$ and $0.70 \mathrm{~mm}$ at $30 \mathrm{~mm}$ depth in azimuth and elevation, respectively. However, an inherent problem of such an array is the FOV, which is limited to the forward looking rectilinear volume region in front of the transducer. For applications such as abdominal and cardiac imaging, a curvilinear volume region is necessary.

Démoré et al. [2] presented a simulation study of a hemispherical shaped 288 element RCA array and compared it to a 128 element linear phased array. To overcome the limited FOV, the array was hemispherical shaped to image a curvilinear volume region of $60^{\circ} \times 60^{\circ}$, while the phased array imaged a sector of $90^{\circ}$. They concluded that RCA arrays could enable a relatively inexpensive volume imaging system capable of real-time volume frame rates $(>20 \mathrm{~Hz})$, with similar contrast, resolution, and dynamic range as the phased array. However, curving the array in both directions is a difficult task from a manufacturing point of view.

A double curved diverging lens in front of the RCA transducer can extend the inherent rectilinear 3-D imaging FOV to a curvilinear volume region [3, 4]. Joyce and Lockwood presented a diverging lens made of RTV-11, cast onto the face of an RCA piezoelectric transducer (PZT) array. The lens material and curvature were optimized using PZFlex (PZFlex, $\mathrm{Cu}-$ pertino, CA, USA), and the resulting lens obtained a FOV of $45^{\circ}$ [3]. Bouzari et al. presented the beamforming method and a simulation study of a diverging lens on a RCA array, and concluded that the limited FOV can be overcome by using a double 


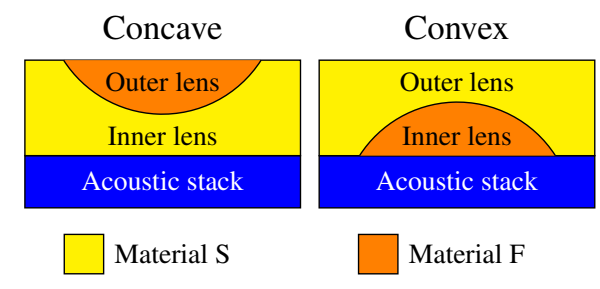

Figure 1: Conceptual sketch of two compound lenses, Concave, and Convex, made from two different materials. The lens type is defined from the shape of the inner lens, i.e. the lens closest to the acoustic stack. For the lens to diverge the beam the speed of sound of material F should be larger than material S.

curved diverging lens [4]. In another paper, Bouzari et al. presented two concave lenses made from room temperature vulcanizing (RTV) rubber fabricated with two different radii, and it was shown that a FOV up to $44.4^{\circ}$ could be obtained [5]. The downside was that lens shapes were concave, since the RTV rubber had a speed of sound lower than tissue. A concave front curvature makes patient contact very difficult as air can be trapped between the patient and the transducer sole, so a flat or convex front is usually a requirement. A lens material with a speed of sound higher than tissue can solve this problem. One concern is that the center of the acoustic wave is attenuated more than the edge, resulting in higher sidelobe levels. Another concern is that these materials are often stiffer, which might be a problem for capacitive micromachined ultrasonic transducers (CMUTs), as this can influence the plate behavior. A different solution could be to use a compound lens of two or more materials to obtain a flat or convex front.

A diverging compound lens was designed and evaluated by Yang et al. for photoacoustic computed tomography [6]. The aim was to increase the receive directivity of the detectors to avoid deformation of off-center targets. They used concave shaped polydimethylsiloxane (PDMS) as the outer layer, and convex shaped epoxy as the inner lens, as illustrated in Fig. 1. It was shown that the $-6 \mathrm{~dB}$ acceptance angle could be increased from $11^{\circ}$ to $55^{\circ}$.

In this paper a compound lens is developed with the main focus on constructing a diverging lens for RCA transducers for increasing the FOV. The $2 N$ channel count of RCA arrays and the increased FOV with a diverging lens offer the potential of 3-D ultrasound imaging, e.g. for cardiac or abdominal applications, in the same price range as conventional 2-D imaging. This can contribute to an increased use of real-time 3-D ultrasound imaging in medical diagnostics, and to the development of new clinical applications.

This paper is organized as follows: Section 2 describes the modeling of a compound lens. A simple analytic model is developed and compared to a finite element method (FEM) model. Section 3 covers the investigation of modifying the acoustic properties of lens materials by loading polymers with inorganic powders. Both decreasing and increasing the speed of sound of the materials are investigated. Section 4 describes the fabrication of a compound add-on lens for a RCA transducer probe, compares the lens behavior to FEM simulations, and demonstrate the possibility of increasing the FOV by imaging a wire phantom. Lastly, a conclusion is presented in Section 5.

\section{Compound Lens Modeling}

A sketch of two types of compound lenses with two lens materials, $\mathrm{F}$ and $\mathrm{S}$, and a flat outer surface is shown in Fig. 1. The lens type is defined from the shape of the inner lens, i.e. the lens closest to the acoustic stack. For the lens to diverge the beam the speed of sound in material $\mathrm{F}$ should be higher than in material S. The following section describes an analytic approach for predicting the focal depth as function of material properties and dimensions, and it is evaluated in relation to a FEM model.

\subsection{Analytic model}

Compound lenses can be analytically modeled using Snell's law, the small angle approximation, and assuming that the lens is infinitely thin. The angle of incidence and refraction of the first boundary, between the inner and the outer lens, is named $\theta_{1}$ and $\theta_{2}$, and at the second boundary, between the outer lens and the medium, is named $\theta_{3}$ and $\theta_{4}$, as shown in Fig. 2. The angles, when the small angle approximation is employed, are

$$
\theta_{1} \approx \frac{L}{2 R}, \quad \theta_{2} \approx \frac{c_{2}}{c_{1}} \theta_{1}, \quad \theta_{3}=\theta_{2}-\theta_{1}, \quad \theta_{4} \approx \frac{c_{m}}{c_{2}} \theta_{3},
$$

where $R$ is the radius of curvature, $L$ is the elevation height, $c_{1}$ is the speed of sound in the inner lens, $c_{2}$ is the speed of sound in the outer lens, and $c_{m}$ is the speed of sound in the medium. The geometric focal depth of the lens, $f_{D}$, is

$$
f_{D} \approx \frac{L}{2 \theta_{4}} .
$$

By combining the above equations, the radius, focal depth, and material speeds of sound can be related by

$$
R=f_{D}\left(\frac{c_{m}}{c_{2}}\right)\left(\frac{c_{2}}{c_{1}}-1\right)=f_{D} \alpha
$$

or in a nondimensional form,

$$
\frac{R}{L}=f_{\#} n_{2}\left(n_{1}-1\right)=f_{\#} \alpha
$$

where $f_{\#}$ is the focusing f-number $\left(f_{\#}=f_{D} / L\right), n_{2}$ is the index of refraction between the outer lens and the medium $\left(n_{2}=\right.$ $c_{m} / c_{2}$ ), and $n_{1}$ is the index of refraction between the inner lens and the outer lens $\left(n_{1}=c_{2} / c_{1}\right)$. (3) and (4) reveals that there is a linear relationship between the focal depth and the radius of curvature, and that the slope, $\alpha$, is controlled by the material properties. This model is valid for both converging and diverging lenses.

\subsection{FEM Model}

FEM models of diverging compound lenses were developed in PZFlex (PZFlex, Cupertino, CA, USA) for the lenses sketched in Fig. 2. A 2-D model with symmetry applied across the center axis at the middle of the lens was utilized. A pressure wave was generated along one of the edges to mimic an 


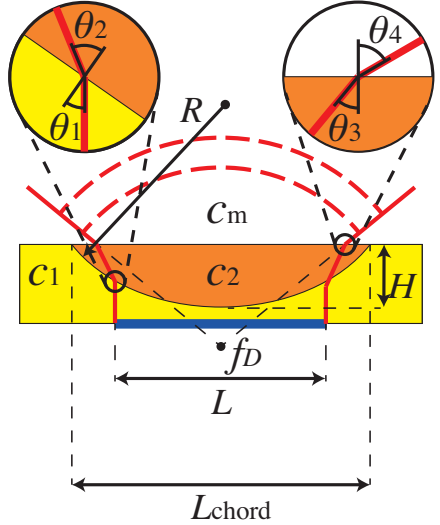

(a) Concave

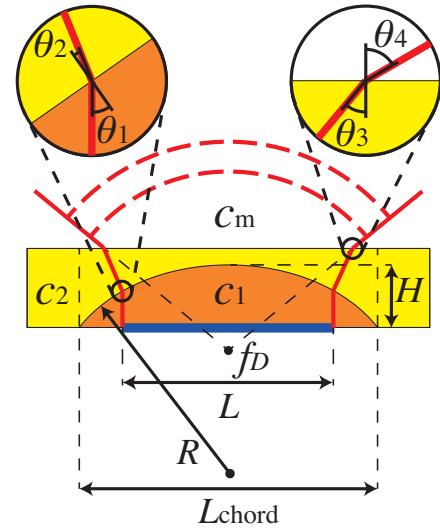

(b) Convex
Figure 2: A compound diverging lens of two materials with different speeds of sound $c_{1}$ and $c_{2}$. Such lenses can be manufactured in (a) a concave $\left(c_{1}<c_{2}\right)$ or (b) a convex $\left(c_{1}>c_{2}\right)$ shape. The edge of the beam is highlighted with a solid red line, and the wave front is shown as a dashed red line. The color scheme is the same as used in Fig. 1, and the blue line indicates the position of the acoustic stack.

emitting transducer with an elevation, $L$, of $20 \mathrm{~mm}$, and it was transmitted through the compound lens and into the medium. The pressure wave was a $3 \mathrm{MHz}$ single cycle sinusoid. Both a convex and a concave lens were simulated. The longitudinal speed of sound of material $\mathrm{S}$ was $0.8 \mathrm{~mm} / \mu \mathrm{s}$, material $\mathrm{F}$ was $2 \mathrm{~mm} / \mu \mathrm{s}$, and the medium was $1.5 \mathrm{~mm} / \mu \mathrm{s}$. The two lens materials and the medium were perfectly matched with respect to specific acoustic impedance, and the acoustic attenuation was neglected. The chord length of the lens, $L_{\text {chord }}$, was $5 \%$ wider than the elevation, $L$, and the height of the arc, $H$, depended on the radius as

$$
H=|R|-\sqrt{R^{2}-\left(\frac{L_{\text {chord }}}{2}\right)^{2}}
$$

The size of the simulation window was $20 \mathrm{~mm}$ wide and $20 \mathrm{~mm}$ high, and a square mesh with an element side length of $5.55 \mu \mathrm{m}$ was used, corresponding to 48 elements per wavelength in material $\mathrm{S}$. An absorbing boundary was used at the edges of the medium. The simulated time period was $14 \mu \mathrm{s}$, allowing enough time for the pressure wave to propagate all the way through the simulation window.

Fig. 3 shows how the calculated pressure fields of a concave and convex compound lens were evaluated to calculate the FOV. The beam profiles were generated by storing the maximum pressure amplitude at each mesh point during propagation of the pressure wave fronts. The $-6 \mathrm{~dB}$ beam width was used to evaluate the FOV, highlighted with a red dashed line, and is related to the f-number, $f_{\#}$, by

$$
f_{\#}=\frac{1}{2} \cot \left(\frac{\mathrm{FOV}}{2}\right)
$$

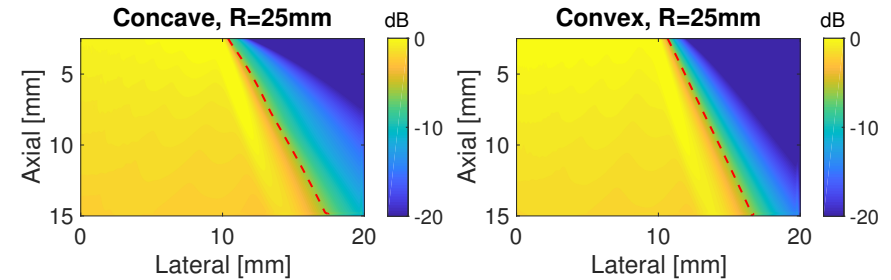

Figure 3: The beam profiles are generated by storing the maximum pressure amplitude at each mesh point during propagation of the pressure wave fronts and are normalized to the excitation pressure amplitude. The $-6 \mathrm{~dB}$ beam width was used to evaluate the FOV and the dashed red line shows the FOV of these two lenses.
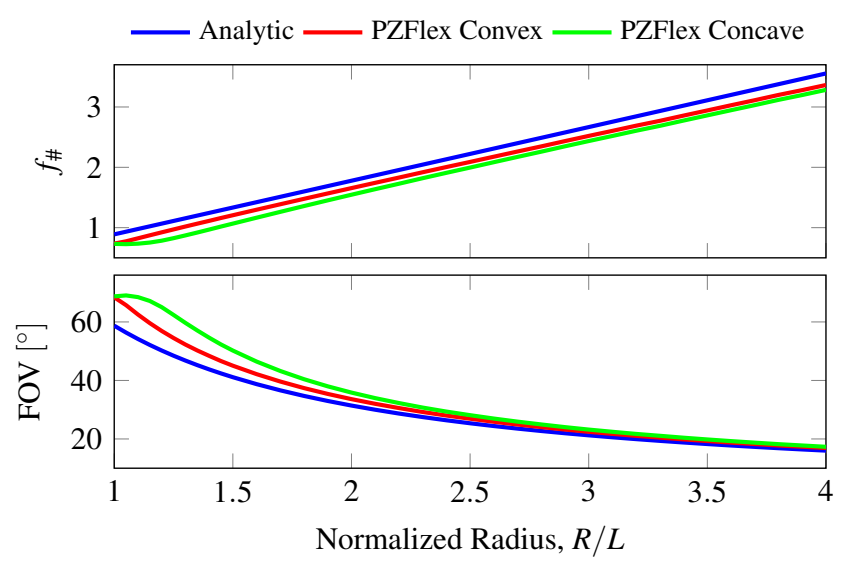

Figure 4: The analytic model and the two PZFlex models are compared with respect to the f-number, $f_{\#}$, and the FOV. In the simulations an elevation of $L=20 \mathrm{~mm}$ was used.

\subsection{Results \& Discussion}

The results of the analytic model and the two PZFlex models, convex and concave, are compared in Fig. 4 for radii between $20 \mathrm{~mm}$ and $80 \mathrm{~mm}$. Notice that the linear relation between the radius and the f-number are observed as expected, and the slopes, $\alpha$, are $\alpha_{\text {analytic }}=2.22, \alpha_{\text {convex }}=2.19$, and $\alpha_{\text {concave }}=2.22$. The relative difference between the slope values are less than $1.5 \%$. Offsets between the three models are observed with the convex model predicting a 0.1 higher f-number than the concave model on average and the analytic model predicting a 0.25 higher f-number on average.

Below a radius of $25 \mathrm{~mm}(R / L=1.25)$, the result of the simulation of the concave lens diverges from the expected linear relation. This was due to internal reflections within the compound lens as seen in Fig. 5. This figure shows the pressure field for a concave lens with a radius of $20 \mathrm{~mm}(R / L=1)$. The interface between the two lens materials and the medium is highlighted with a red line. Part of the pressure field was reflected from the interface of the two lens materials and was propagating within the lens.

The critical radius of the lens, $R_{\mathrm{c}}$, for internal reflections in the lens can be estimated using the criterion for total internal reflection. Total reflection occurs when 


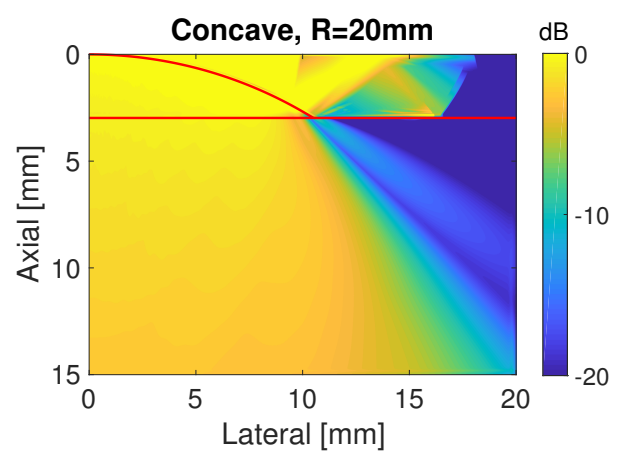

Figure 5: The internal reflection of the concave lens type with a normalized radius of $R / L=1$. The red lines show the interface between the different materials of the lens and the medium.

$$
\frac{\sin \left(\Theta_{c}\right)}{c_{1}}=\frac{\sin \left(90^{\circ}\right)}{c_{2}} \Rightarrow \Theta_{c}=\arcsin \left(\frac{c_{1}}{c_{2}}\right) .
$$

The angle of the lens at the edge of the array is determined by the geometry of the lens as

$$
\Theta=\arcsin \left(\frac{L}{2 R}\right) .
$$

The critical radius, $R_{\mathrm{c}}$, is found by combining the two above equations and setting $R=R_{\mathrm{c}}$ and $\Theta=\Theta_{\mathrm{c}}$ :

$$
R_{\mathrm{c}}=\frac{L}{2} \frac{c_{2}}{c_{1}} .
$$

The critical radius depends both on the ratio of the speed of sound of the lens materials and on the elevation of the emitting transducer. Inserting the speeds of sound and the dimensions used in the simulation, we find that the critical radius is $25 \mathrm{~mm}$ $(R / L=1.25)$, which is in perfect agreement with the observed simulation results. Notice that this model is simulating the lens effect during emission. During reception, the convex lens will have the same problem, but will instead limit the angular receive response.

\section{Composite Materials}

From the above analysis, based on 4, it is evident that higher speed of sound ratios, $n_{1}$ and $n_{2}$, result in a larger FOV. This section will therefore describe how the speed of sound of materials can be altered by loading a base material with powder to obtain a 0-3 composite material. First a model is introduced to predict the speed of sound in a composite material, and then four different composites are investigated, one designed to decrease the speed of sound and three designed to increase the speed of sound. The experimental results are then compared to the model.

\subsection{Devaney model}

The Devaney model [7] was used to calculate the effective elastic properties of the $0-3$ composites used in this study. This model was used as it could predict the speed of sound within $6 \%$ on average compared to experiments and was the only model able to predict the behavior above a volume concentration, $C$, of $40 \%$ as seen by Nguyen et. al. [8]. The model is based on multiple-scattering theory and assumes a random distribution of identical spherical elastic inclusions embedded in an infinite isotropic elastic matrix. In addition, the wavelength of the ultrasonic wave in the composite is assumed to be much larger than the particle size. This yields two coupled equations to calculate the effective bulk, $K$, and shear moduli, $G$ :

$$
\begin{aligned}
& K=K_{1}+C \frac{(3 K+4 G)\left(K_{2}-K_{1}\right)}{3 K+4 G+3\left(K_{2}-K_{1}\right)} \\
& G=G_{1}+C \frac{5(3 K+4 G) G\left(G_{2}-G_{1}\right)}{(15 K+20 G) G+6(K+2 G)\left(G_{2}-G_{1}\right)}
\end{aligned}
$$

where $K_{1}$ is the bulk modulus of the base, $G_{1}$ is the shear modulus of the base, $K_{2}$ is the bulk modulus of the dopant/inclusion, $G_{2}$ is the shear modulus of the dopant/inclusion, and $C$ is the volume concentration of the inclusions. The two coupled equations are solved numerically for the elastic constants, and the iteration process is terminated when changes in the moduli are less than $0.01 \%$ from one iteration to the next. The effective density of the composite is simply the volume weighted average of the medium and its inclusions:

$$
\rho=\rho_{1}+C\left(\rho_{2}-\rho_{1}\right),
$$

where $\rho$ is the density of the composite, $\rho_{1}$ is the density of the base, and $\rho_{2}$ is the density of the inclusion. The longitudinal speed of sound of the composite is then calculated by

$$
c_{l}=\sqrt{\frac{K+\frac{4}{3} G}{\rho}} .
$$

A high speed-to-density ratio material should be used to increase the speed of sound, whereas a material with a low ratio should be used to decrease the speed of sound.

\subsection{Experimental Composites}

Two commercially-available, unfilled 2-part base materials (part A: base and part B: curing agent) were used in this study, an RTV silicone rubber, RTV615 (Momentive Performance Materials Inc., Waterford, NY, USA ) and an urethane, Hapflex 541 (Hapco Inc., Hanover, MA, USA). Both materials were low viscosity resins, which was desirable for ease of powder loading, degassing, and mold filling. Three different powders were tested, AC6111 Boron Nitride (Momentive Performance Materials Inc., Waterford, NY, USA), referred to as BN, iM30K glass bubbles (3M, Maplewood, MN, USA), referred to as iM30K, and bismuth oxide nanopowder (US Research Nanomaterials Inc., Houston, TX, USA), referred to as $\mathrm{Bi}_{2} \mathrm{O}_{3}$. The material properties of both the base materials and the powders are listed in Table 1.

$\mathrm{BN}$ and $\mathrm{iM} 30 \mathrm{~K}$ were selected for their high speed-to-density ratios, 7.1 and 6.6, respectively, and $\mathrm{Bi}_{2} \mathrm{O}_{3}$ was selected for its 
Table 1: Material properties of base materials and powders, density $\rho$, longitudinal speed of sound $c_{l}$, shear speed of sound $c_{s}$, and the particle size.

\begin{tabular}{l|cccc}
\hline \hline Material & $\begin{array}{c}\rho \\
{\left[\mathrm{g} / \mathrm{cm}^{3}\right]}\end{array}$ & $\begin{array}{c}c_{l} \\
{[\mathrm{~mm} / \mu \mathrm{s}]}\end{array}$ & $\begin{array}{c}c_{s} \\
{[\mathrm{~mm} / \mu \mathrm{s}]}\end{array}$ & $\begin{array}{c}\text { Size } \\
{[\mu \mathrm{m}]}\end{array}$ \\
\hline RTV 615 & 1.02 & 1.03 & 0.03 & - \\
Hapflex 541 & 1.05 & 1.52 & 0.1 & - \\
$\mathrm{Bi}_{2} \mathrm{O}_{3}$ & 8.9 & 4.79 & 2.41 & $0.08-0.2$ \\
$\mathrm{BN}$ & 2.1 & 15 & 10 & 0.5 \\
$\mathrm{iM} 30 \mathrm{~K}$ & 0.6 & 3.96 & 1.24 & 18 \\
\hline \hline
\end{tabular}

low speed-to-density ratio, 0.5 . From a variety of low speedto-density ratio materials, $\mathrm{Bi}_{2} \mathrm{O}_{3}$ was chosen for its apparent minimal attenuation [9].

Three different combinations were investigated to obtain a composite material with an increased speed of sound: RTV615 + BN, RTV615 + iM30K and Hapflex 541 + iM30K, and one combination was investigated to decrease the speed of sound: RTV615 $+\mathrm{Bi}_{2} \mathrm{O}_{3}$.

The $\mathrm{Bi}_{2} \mathrm{O}_{3}$ was bought in a $\beta$-phase, but this phase showed to act as a cure inhibitor for the RTV615 silicone rubber. This problem was previously described in [10]. The $\mathrm{Bi}_{2} \mathrm{O}_{3}$ was therefore pretreated by heating the powder up to $450^{\circ} \mathrm{C}$ for two hours and then it was slowly cooled down to room temperature over at least five hours. This changed the phase of the $\mathrm{Bi}_{2} \mathrm{O}_{3}$ from a tetragonal $\beta$-phase to a monoclinic $\alpha$-phase. The $\alpha$ phase powder does not act as a cure inhibitor for the RTV615.

The BN and iM30K were mixed into part A of the base materials by hand and then part $\mathrm{B}$ was mixed in the composite also by hand. The particle size of the $\mathrm{Bi}_{2} \mathrm{O}_{3}$ was $80-200 \mathrm{~nm}$ and therefore, to reduce the effect of agglomeration of the nanometer sized particles, the powder was mixed into part A using a planetary centrifugal Mixer, Thinky Mixer AR-100 (Thinky, Tokyo, Japan), and ten $3 \mathrm{~mm}$ zirconia balls were added to the composite. The balls were used for increasing the shear force during mixing to de-agglomerate the nano particles. This was done five times for two minutes at $2000 \mathrm{rpm}$ with three minutes of cooling between each. The ten zirconia balls were then removed and part B was mixed into the composite for two minutes at $2000 \mathrm{rpm}$. The composite was then degassed under vacuum for three minutes and three samples were made out of each composite with a diameter of $25 \mathrm{~mm}$ and a thickness of $2 \mathrm{~mm}, 3 \mathrm{~mm}$ and $4 \mathrm{~mm}$. The samples were then cured overnight at $45^{\circ} \mathrm{C}$.

\subsection{Measurement setup}

Density measurements were made with a Mettler Toledo XP204 (Mettler Toledo, Columbus, OH, USA) using their density kit. Each sample was measured three times and the mean was calculated across the three samples.

The speed of sound and the acoustic attenuation of the longitudinal wave were measured based on a pulse throughtransmission method in water using two identical $5 \mathrm{MHz}$ transducers placed at a distance of $8 \mathrm{~cm}$ from each other. A pulser/receiver module (Model 5800, Panametrics Corp., Waltham, MA, USA) was used to generate a broad band pulse with an energy of $12.5 \mu \mathrm{J}$ and a damping of $50 \Omega$. The transmitted pulse had a $12 \mathrm{~dB}$ bandwidth of $6.3 \mathrm{MHz}$ centered around

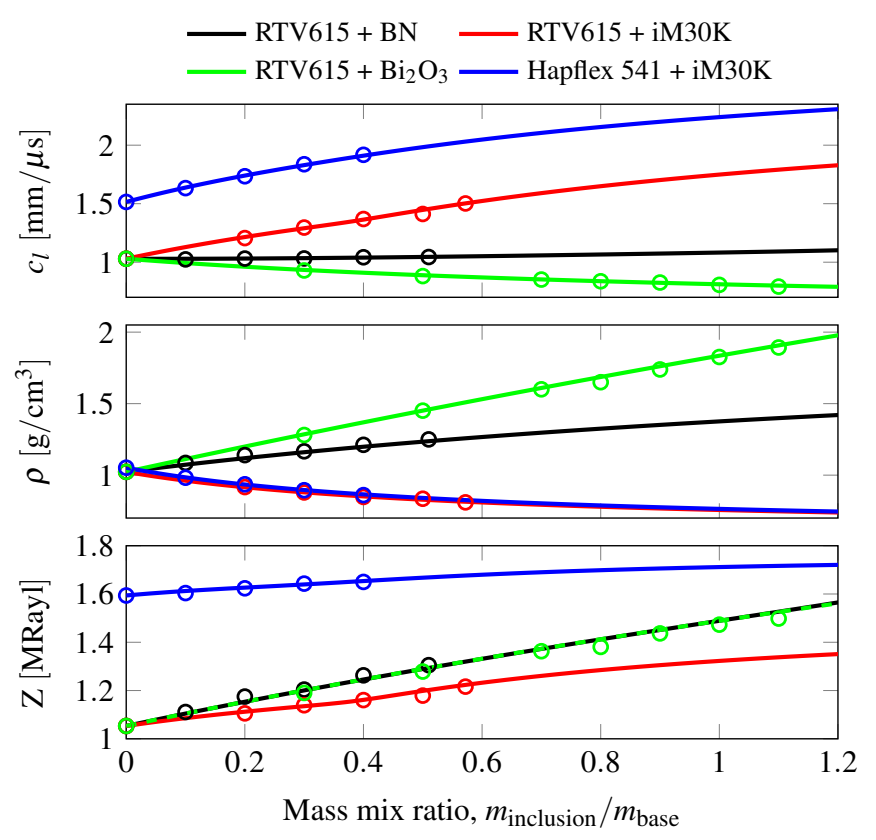

Figure 6: The specific acoustic impedance, density, and longitudinal speed of sound for different mass mix ratios have been measured (circles on the graphs) and compared to the analytical Devaney model (solid lines on the graph).

$5 \mathrm{MHz}$. The output waveform of the second transducer was transmitted from the pulser/receiver module to a digital oscilloscope (9314CM, LeCroy Corp., Chestnut Ridge, NY, USA) through a $50 \Omega$ coaxial cable for digitization. A frequency domain analysis was performed, as described in [11], yielding the longitudinal speed of sound and attenuation. The speed of sound reported is an average of the calculated speed of sound in the frequency interval 2.5 and $7.5 \mathrm{MHz}$, as this was within the $12 \mathrm{~dB}$ bandwidth, and the attenuation was calculated at a frequency of $5 \mathrm{MHz}$.

\subsection{Results \& Discussion}

Fig. 6 shows the longitudinal speed of sound, $c_{l}$, density, $\rho$, and the specific acoustic impedance, $Z$, for varying mass mix ratios (MMRs), $m_{\text {inclusion }} / m_{\text {base }}$, for all four composite materials. The measured values are represented with circles on the graphs, and the analytic Devaney model is compared to the measurements and are represented with solid lines. The measured values are in agreement with the calculated curves within $2.5 \%$, both with respect to density and speed of sound.

The amount of powder loaded into the base material was limited for both the BN and the iM30K powder because the viscosity increased to an extent where it was not possible to degas the composite or fill the mold used for creating the acoustic samples. A non-Newtonian behavior, a shear thinning effect, of the $\mathrm{BN}$ mixtures was observed, so a lower volume concentration could be obtained compared to the composites containing iM30K. The largest mass mix ratio obtained of RTV615 + BN was $M M R=0.51$ corresponding to a volume concentration of $C=19.9 \%$. For RTV615 $+\mathrm{iM} 30 \mathrm{~K}$ it was $M M R=0.57$ corre- 

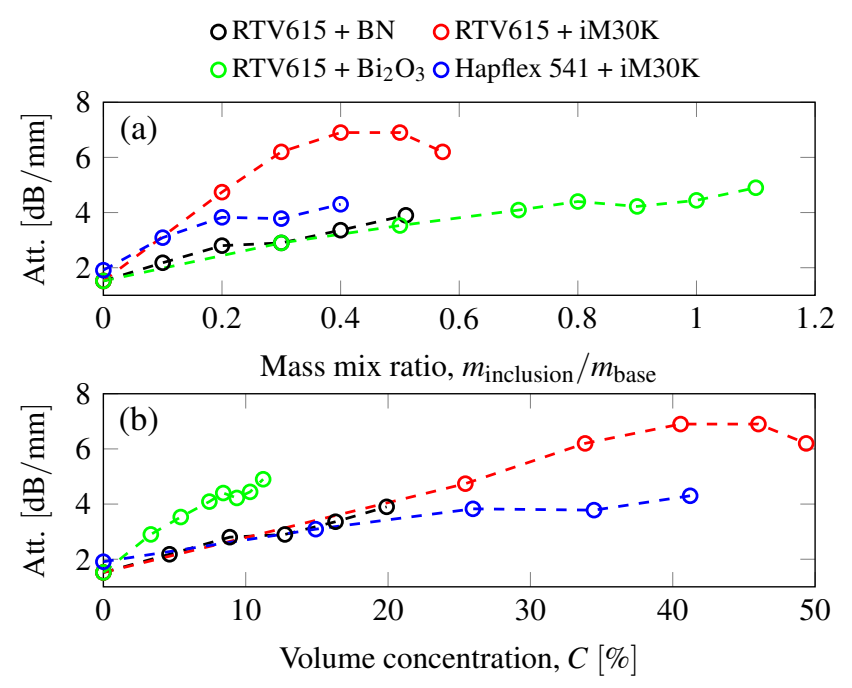

Figure 7: Attenuation at 5MHz. The circles represents the measured values and the dashed lines are only plotted as a visual aid.

sponding to $C=49.4 \%$, and for Hapflex $541+\mathrm{iM} 30 \mathrm{~K}$ it was $M M R=0.4$ corresponding to $C=41.2 \%$.

BN was investigated for increasing the speed of sound in this study, but the density was still larger than the density of the two base materials. Loading the RTV615 with the maximum amount of $\mathrm{BN}$, the speed of sound only increased from $1.03 \mathrm{~mm} / \mu$ s to $1.04 \mathrm{~mm} / \mu \mathrm{s}$, an increase of $1.4 \%$. However, loading the RTV615 with iM30K was much more effective as the maximum amount increased the speed of sound to $1.50 \mathrm{~mm} / \mu \mathrm{s}$, an increase of $46 \%$. Loading the Hapflex 541 with the maximum amount of $\mathrm{iM} 30 \mathrm{~K}$ increased the speed of sound from $1.52 \mathrm{~mm} / \mu \mathrm{s}$ to $1.93 \mathrm{~mm} / \mu \mathrm{s}$, an increase of $27 \%$. Loading the RTV615 with $\mathrm{Bi}_{2} \mathrm{O}_{3}$ at $M M R=1.1$, corresponding to $C=11.2 \%$, decreased the speed of sound from $1.03 \mathrm{~mm} / \mu \mathrm{s}$ to $0.792 \mathrm{~mm} / \mu \mathrm{s}$, a decrease of $30 \%$.

It is not only the density and speed of sound that changes with the MMR; the acoustic attenuation is also affected. The measured acoustic attenuation of the longitudinal wave at $5 \mathrm{MHz}$ for varying MMR is shown in Fig. 7(a) and the volume concentration, $C$, in Fig. 7(b). The circles represent the measured values and the dashed lines are only plotted as a visual aid. As expected, the attenuation increases with the volume concentration of the powder in the composite material, and up to around $20 \%$ the relation seems to be linear. The slopes and the $95 \%$ confidence intervals are listed in Table 2 . The RTV615 $+\mathrm{Bi}_{2} \mathrm{O}_{3}$ has a significant steeper slope than the three other composites at a 95\% confidence level, as their is no overlap in the confidence intervals. This indicates that the losses are driven primarily by the density of the inclusion. This presented a dilemma when the composite materials speed of sound was lowered, since a higher particle density was wanted in order to affect the speed of sound and the specific acoustic impedance with low volume concentrations, but this in turn had the greatest contribution to the attenuation. The particle size and density were not of great concern with respect to attenuation when a powder was chosen for increasing the speed of sound, which is also seen Table 2,
Table 2: Slopes and the corresponding confidence interval at a 95\% level of the volume concentration versus attenuation of the composites.

\begin{tabular}{l|ccc}
\hline \hline & Slope & \multicolumn{2}{c}{ Confidence interval $[\mathrm{dB} / \mathrm{mm} / \%]$} \\
Composite material & {$[\mathrm{dB} / \mathrm{mm} / \%]$} & Lower bound & Upper bound \\
\hline RTV 615+BN & 0.1126 & 0.0892 & 0.1359 \\
RTV 615+iM30K & 0.1344 & 0.1108 & 0.1580 \\
Hapflex 541 + iM30K & 0.07431 & 0.0301 & 0.1182 \\
RTV 615+Bi $2 \mathrm{O}_{3}$ & 0.2799 & 0.2210 & 0.3388 \\
\hline \hline
\end{tabular}

as the composites containing $\mathrm{BN}$ and $\mathrm{iM} 30 \mathrm{~K}$ did not show any significant difference in attenuation dependence on the volume concentration.

\section{Diverging lens}

A doubled-curved diverging compound add-on lens was fabricated and experimentally evaluated in this section. The addon lens gave the possibility of using the lens as either a concave lens or as a convex depending on which side was facing the RCA transducer.

\subsection{Lens Design}

The design and materials used for the diverging lens were based on the findings in the two previous sections. To maximize the energy transfer from the acoustic stack to the tissue, the total attenuation of the lens should be minimized. Both the total thickness of the lens and the composite material attenuation will affect this. The minimum thickness was determined by the arc height between the two lens materials. (5) shows that a large radius, $R$, was desired as this decreased the arc height, $H$. For a fixed focal depth, $f_{D}$, increasing the speed of sound ratio of the outer to the inner lens material, $c_{2} / c_{1}$, and of the medium (water/tissue) to the outer lens material $c_{m} / c_{2}$, increases the radius and thereby decreases the arc height.

As two materials were used, the net attenuation can vary across the elevation, contributing to the apodization of the beam. It is preferable to have more attenuation at the edges than at the center of the elevation. The degree of apodization was contingent on both the attenuation of the constituent materials and their relative geometry, specifically, the arc height between them.

Another factor in maximizing the energy transfer between the acoustic stack and the tissue was the specific acoustic impedance matching between the lens materials and the tissue. The intensity transmission coefficient, $T$, and reflection coefficient, $R$, are specified by the following formulas [12]

$$
\begin{aligned}
& T=\frac{4 \frac{Z_{2}}{Z_{1}}}{\left(\frac{Z_{2}}{Z_{1}}+1\right)^{2}} \\
& R=1-T,
\end{aligned}
$$

where $Z_{1}$ and $Z_{2}$ are the specific acoustic impedances of the two materials. These formulas are only valid for plane waves 


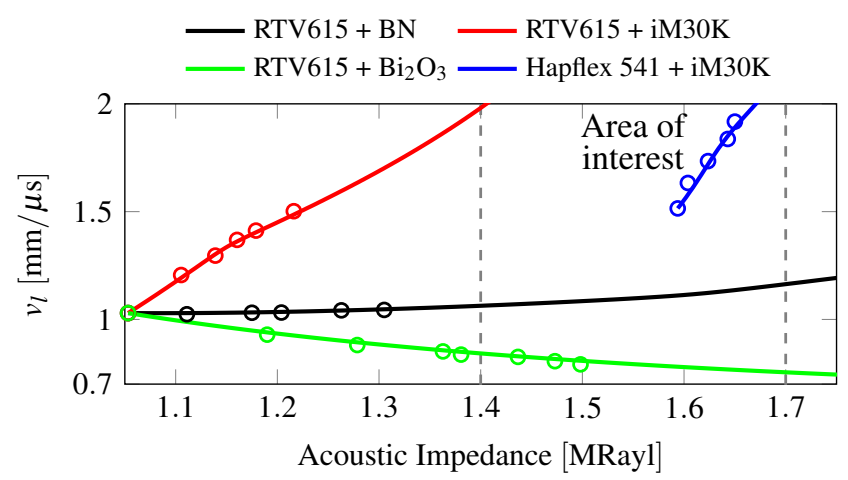

Figure 8: The measured (circles) and calculated (solid line) longitudinal speeds of sound plotted as a function of specific acoustic impedance for the four different composites. The area of interest is chosen to obtain a reflected power of less than $25 \mathrm{~dB}$ below the original power from the interface to tissue.

Table 3: Material properties for the compound diverging lens. The attenuations are reported at $5 \mathrm{MHz}$.

\begin{tabular}{l|cccc}
\hline \hline Composite material & $\begin{array}{c}\text { MMR } \\
-\end{array}$ & $\begin{array}{c}\text { Long. speed } \\
{[\mathrm{mm} / \mu \mathrm{s}]}\end{array}$ & $\begin{array}{c}\mathrm{Z} \\
{[\mathrm{MRayl}]}\end{array}$ & $\begin{array}{c}\text { Att. } \\
{[\mathrm{dB} / \mathrm{mm}]}\end{array}$ \\
\hline RTV 615+ $\mathrm{Bi}_{2} \mathrm{O}_{3}$ & 0.9 & 0.826 & 1.45 & 4.42 \\
Hapflex 541 & 0 & 1.52 & 1.59 & 1.91 \\
\hline \hline
\end{tabular}

impinging orthogonally on planar boundaries so they do not apply directly for this situation, but serve to provide guidelines for specific acoustic impedance matching goals.

For specific acoustic impedance ratios under 1.35, the loss due to reflections amounts only to about $0.1 \mathrm{~dB}$ each way. The reflected power at this specific acoustic impedance ratio is about $16.5 \mathrm{~dB}$ below the original power, which may be at a sufficiently high level to cause concern about unwanted reverberations. To obtain a reflected power of less than $25 \mathrm{~dB}$ below the original power requires that the specific acoustic impedance ratio is lower than 1.1. For tissue with a specific acoustic impedance of 1.54 MRayl this corresponds to an area of interest between 1.4 MRayl and 1.7 MRayl. The specific acoustic impedance ratio between the two lens materials should also be lower than 1.1 .

The above analysis gives a set of requirements for the materials used for the lens. The measured (circles) and calculated (solid line) longitudinal speeds of sound are plotted in Fig. 8 as a function of specific acoustic impedance for all the composite materials. Only Hapflex $541+\mathrm{iM} 30 \mathrm{~K}$ and RTV615+ $\mathrm{Bi}_{2} \mathrm{O}_{3}$ lie within the region of interest. As the attenuation of the composite material is proportional to the volume percent of the powder, Hapflex 541 was used as material $\mathrm{F}$ without loading to minimize attenuation. This selection sets a minimum acceptable specific acoustic impedance of 1.45 MRayl for material S. Again, to reduce the attenuation, $\mathrm{RTV} 615+\mathrm{Bi}_{2} \mathrm{O}_{3}$ with the lowest MMR within the limit was used: $1: 0.9$. The material properties of the two materials used for the lens are listed in Table 3.

Using RTV $615+\mathrm{Bi}_{2} \mathrm{O}_{3}$ and Hapflex 541, and an active transducer aperture of $16.74 \times 16.74 \mathrm{~mm}^{2}$, the critical radius, hence the minimum radius of the lens, becomes $21.7 \mathrm{~mm}$. A radius of the lens of $25.4 \mathrm{~mm}$ was then chosen to avoid internal reflection even with a $10 \%$ variation of the speed of sound. This gives a theoretical f-number of 1.86, corresponding to an FOV of $30.1^{\circ}$. To cover the whole transducer, including the integrated hardware apodization, the chord length becomes $L_{\text {chord }}=27.3 \mathrm{~mm}$, which results in the fabricated lens having an arc height of $H=4 \mathrm{~mm}$ of the curved part of the lens and a total thickness of $4.7 \mathrm{~mm}$. The one-way attenuation at $5 \mathrm{MHz}$ at the center and at the edge is therefore $9.9 \mathrm{~dB}$ and $18.9 \mathrm{~dB}$, respectively. The attenuation is a challenge, especially for diverging lenses as a low f-number and high FOV are desirable resulting in a thick lens. However, RCA arrays may be less susceptible to the high attenuation through the lens, since their element size (and therefore transmitted or received energy) is more than twice as large as comparable 1-D arrays.

\subsection{Lens Fabrication}

A mold of RTV silicone, RTV664 (Momentive Performance Materials Inc., New York, USA), was cast into a rigid plastic frame, and a curved surface was formed using a stainless steel ball bearing with a radius of $25.4 \mathrm{~mm}$. A mold assembly was made, consisting of a flat bottom plate and a top plate with a circular hole in which a steel ball sat during curing. The frame was sandwiched between these two plates. During cure, pressure was applied to push the ball down into the mold assembly. After curing, the ball was removed and the excess RTV664 was cut away with a scalpel.

A Hapflex 810 primer (Hapco Inc., Hanover, MA, USA) was applied to the inner part of the rigid plastic frame with a qtip, the excess primer was blown away with a nitrogen gun, and the remaining primer was allowed to set for two minutes. Hapflex 541 was poured into the rigid plastic frame with the RTV664 mold in it. It was degassed in vacuum for two minutes, and sandwiched between two flat plates. The two plates were held together with binder clips and the Hapflex 541 was cured overnight at room temperature in a nitrogen atmosphere at $375 \mathrm{kPa}$ to help reduce voids as suggested by the manufacturer.

After curing, the RTV664 mold was removed from the frame as shown in Fig. 9(a), and a platinum primer, A-306 (Factor II, Inc., Arizona, USA), was applied to the inner part of the frame and the Hapflex 541. The primer was dried in a humidity chamber at room temperature for one hour to actuate it. RTV615 $+\mathrm{Bi}_{2} \mathrm{O}_{3}$, in a MMR of 1:0.9, was then poured onto the Hapflex 541 in the plastic frame, degassed in vacuum for three minutes, sandwiched between two flat plates, and held together with binder clips. The RTV615 $+\mathrm{Bi}_{2} \mathrm{O}_{3}$ was cured overnight at $45^{\circ} \mathrm{C}$. The finished add-on lens seen from the Hapflex 541 side is shown in Fig. 9(b).

\subsection{Measurement setup}

To evaluate the performance of the diverging compound lens, a PZT RCA 62+62 element 2-D array was used, which is described in [13]. The lens was placed in front of the probe using a specialized holder as shown in Fig. 10 and described in [5], with water as a coupling media between the lens and the transducer. 


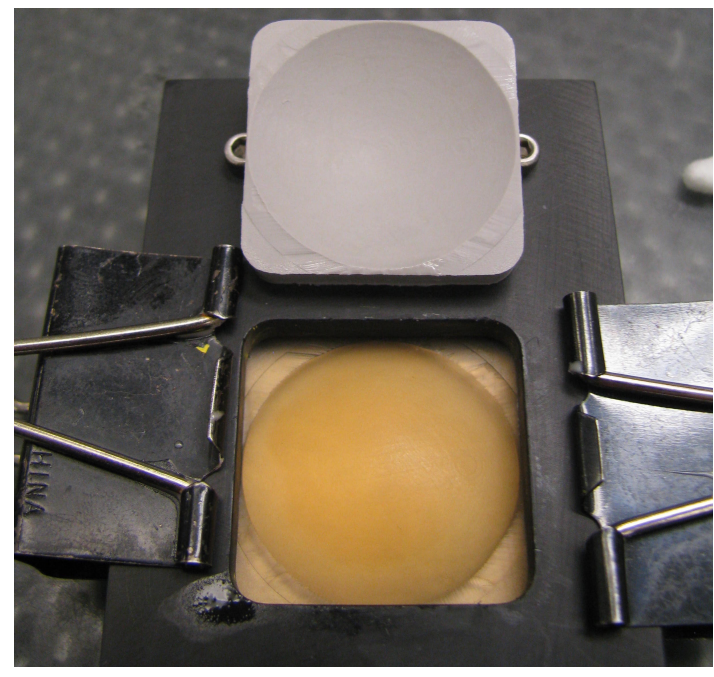

(a)

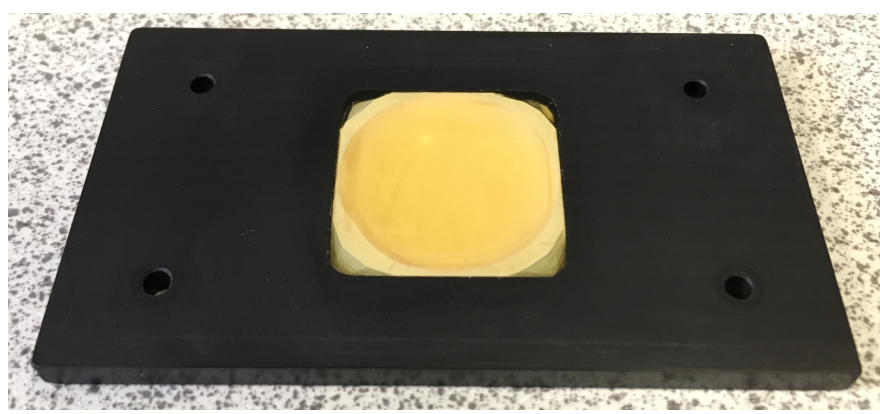

(b)

Figure 9: Fabrication of the add-on lens. (a): After removal of the gray colored RTV664, which is used as a mold for the Hapflex 541. (b): The finished add-on lens seen from the Hapflex 541 side.

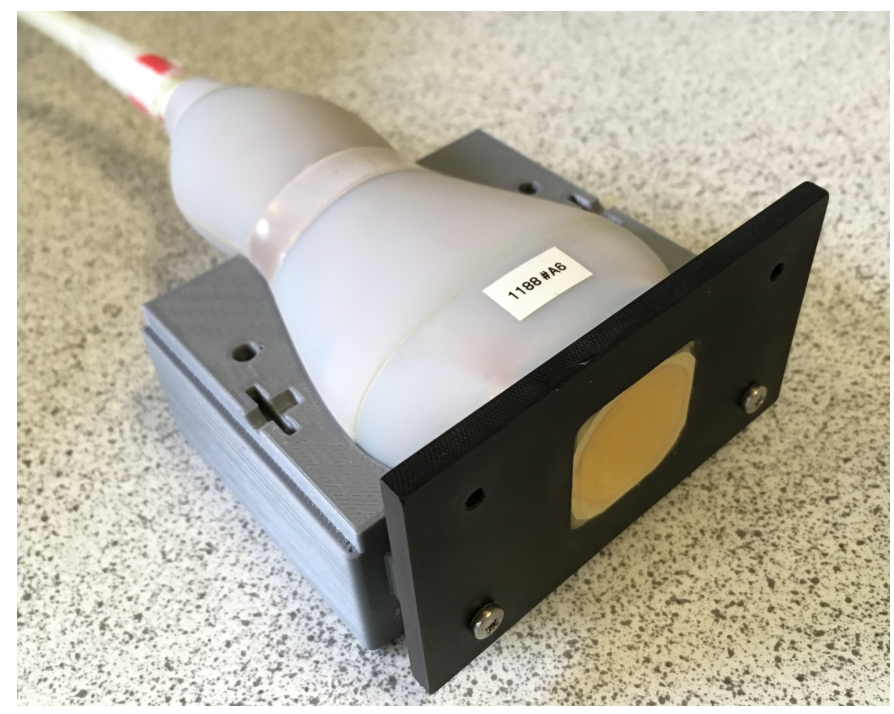

Figure 10: The lens module was placed in front of the probe using a holder (half of the holder is shown in the figure).
The transmit pressure measurements of the lens were carried out using an AIMS III intensity measurement system (Onda Corp., California, USA) with an Onda HGL-0400 Hydrophone connected to the experimental research ultrasound scanner, SARUS [14]. All elements were excited at the same time with a 1-cycle $3 \mathrm{MHz}$ sinusoidal pulse and the hydrophone was moved in a $56 \mathrm{~mm}$ by $17.5 \mathrm{~mm}$ grid in front of the transducer with a pitch of $0.05 \mathrm{~mm}$ in both directions and recording the pressure in the center lateral plane with a sampling frequency of $70 \mathrm{MHz}$. At each position five measurements were recorded and the mean was calculated to average out of the noise of the system. Because the hydrophone was moved in a Cartesian coordinate system the directivity should be considered to compensate the measured signal. As referred to by the hydrophone manufacture, ONDA, the directivity of an unbaffled hydrophone can be estimated by $[15,16]$

$$
D(\theta)=\frac{1+\cos \theta}{2} \frac{2 J_{1}(k a \sin \theta)}{k a \sin \theta}
$$

where $D$ is the directivity function (measured pressure of the hydrophone normalized to the measured pressure of a normal incident wave), $\theta$ is the angle relative to the normal of the hydrophone surface, $J_{1}$ is the Bessel function of first kind of first order, $k$ is the wave number, and $a$ is the radius of the hydrophone aperture, which was $200 \mu \mathrm{m}$ for this specific hydrophone. To estimate the angle, $\theta$, the angle between the focal point to the measurement point and the hydrophone was calculated. The initial focal depth was estimated from the simulation and was placed behind the transducer, and are related to the FOV as stated in (6). By an iterative process the focal depth was updated using the estimated focal depth of the measurement, hence a new angle was calculated for each point, and a new beam profile, FOV and focal depth were calculated. This iteration process was terminated when the change of the FOV from one iteration to the next was less than $0.01 \%$. The hydrophone signal was then scaled at each position in the grid according to (16).

A synthetic aperture imaging (SAI) sequence was designed for imaging down to $14 \mathrm{~cm}$ of depth. It utilized single element transmissions on the row elements, and the echoes were collected with all the column elements. The elements were excited with a 2-cycle sinusoidal pulse with a center frequency of $3 \mathrm{MHz}$. For a speed of sound of $1540 \mathrm{~m} / \mathrm{s}, 182 \mu \mathrm{s}$ was required to acquire a single image line to a depth of $14 \mathrm{~cm}$ including the lens. For 62 emissions this was equivalent to a volume rate of $88 \mathrm{~Hz}$. This was the same sequence that was used in [5]. IQ-modulated RF data were used for beamforming a lowresolution volume for every emission and finally, by summing all the low-resolution volumes in phase, a high-resolution volume was generated. The measured IQ-modulated RF signals were beamformed using a MATLAB (MathWorks Inc., Massachusetts, USA) implementation of the delay-and-sum (DAS) beamformer specific to curved RCA arrays and described in [4] using a FOV of $31^{\circ}$ to calculate the time delay profile for the transducer. 


\subsection{Beam Divergence}

At each measurement position, the maximum pressure amplitude was recorded, and the measured beam profiles of both lens configurations are shown in Fig. 11 together with the simulated beam profiles. The pressure is normalized to the maximum pressure amplitude at each depth, therefore, a constant amplitude is seen at the center for both the measurements and the simulations. The $-6 \mathrm{~dB}$ contour lines are shown as dashed red lines and are used to calculate the FOV. The measured FOV are $32.2^{\circ}$ and $30.4^{\circ}$ for the concave and convex type, respectively. The simulated FOVs are $33.4^{\circ}$ and $33.0^{\circ}$ for the concave and convex lenses, which are $3.7 \%$ and $8.6 \%$ higher than the measured. One effect contributing to the lower measured FOV is that there is no out of plane diffraction/divergence of the beam in the 2-D simulation, whereas that is the case for the measurements, as it is a 3-D system and the lens is curved in both directions.

\subsection{Imaging}

To illustrate the increased FOV obtained using the diverging compound lens, Fig. 12 shows a wire grid phantom, with a diameter of the wires of $0.15 \mathrm{~mm}$, imaged with (a) and without (b) the convex lens. Both figures show a cross-plane in azimuth through the wires down to a depth of $14 \mathrm{~cm}$ and with a dynamic range of $40 \mathrm{~dB}$. Without the lens, Fig. 12(a), only the three center columns of the wire phantom are visible. The wires outside the three center columns are to some extent possible to locate, but the amplitudes are more than $25 \mathrm{~dB}$ lower than the center wires. With the convex lens, Fig. 12(b), the wires within the FOV are all visible with an identical amplitude at the same depth. However, notice that the signal amplitude of the beamformed wires decrease with depth, which is due to the divergence of the energy allowing the increased FOV.

\section{Conclusion}

This paper investigated compound lenses for ultrasound transducers to obtain a flat sole. An analytical and a FEM model of compound lenses have been developed, and they are in agreement with respect to the f-number within $1.5 \%$. A critical radius of the compound lens exists, where internal reflection within the lens will occur, and this radius can easily be found from a simple analytic calculation. A low speed of sound-to-density powder, $\mathrm{Bi}_{2} \mathrm{O}_{3}$, was used to decrease the speed of sound of an $\mathrm{RTV}$, from $1.03 \mathrm{~mm} / \mu$ s down to $0.792 \mathrm{~mm} / \mu \mathrm{s}$. Increasing the speed of sound requires a powder with a high speed of soundto-density ratio. $\mathrm{BN}$ only increased the speed of sound up to $1.4 \%$, hence complex powders, like micro-balloons (iM30K), were investigated. Using $\mathrm{iM} 30 \mathrm{~K}$, the speed of sound of the RTV and the Hapflex 541 was increased from $1.03 \mathrm{~mm} / \mu \mathrm{s}$ to $1.50 \mathrm{~mm} / \mu \mathrm{s}$ and from $1.52 \mathrm{~mm} / \mu \mathrm{s}$ to $1.93 \mathrm{~mm} / \mu \mathrm{s}$, respectively. Using a diverging compound lens with RCA transducers is shown to be a feasible method for increasing the FOV to a curvilinear volume region in front of the transducer with a FOV of $31^{\circ}$. To demonstrate the imaging capability, a wire phantom was imaged down to a depth of $14 \mathrm{~cm}$, and the wires not directly in front of the transducer and within the FOV were visible. Challenges still exist with concern to acoustic attenuation of materials and the thickness of the compound lens. Compound lenses will always be thicker than corresponding single material lenses, due to minimum constituent material thickness requirements. Consequently, alternatives to cylindrical/spherical shapes are attractive because alternate shapes can reduce overall lens thickness.

\section{Acknowledgments}

This work was financially supported by the Danish National Advanced Technology Foundation (82-2012-4) and by BK Ultrasound.

\section{References}

[1] C. H. Seo, J. T. Yen, A 256 x 256 2-D array transducer with row-column addressing for 3-D rectilinear imaging, IEEE Trans. Ultrason., Ferroelec., Freq. Contr. 56 (4) (2009) 837-847.

[2] C. E. M. Démoré, A. Joyce, K. Wall, G. Lockwood, Real-time volume imaging using a crossed electrode array, IEEE Trans. Ultrason., Ferroelec., Freq. Contr. 56 (6) (2009) 1252-1261.

[3] A. W. Joyce, G. R. Lockwood, Crossed-array transducer for real-time 3D imaging, in: Proc. IEEE Ultrason. Symp., 2014, pp. 2116-2120. doi:10.1109/ULTSYM.2014.0527.

[4] H. Bouzari, M. Engholm, C. Beers, M. B. Stuart, S. I. Nikolov, E. V. Thomsen, J. A. Jensen, Curvilinear 3-D imaging using rowcolumn-addressed 2-D arrays with a diverging lens: Feasibility study, IEEE Trans. Ultrason., Ferroelec., Freq. Contr. 64 (6) (2017) 978-988. doi:10.1109/TUFFC.2017.2687521.

[5] H. Bouzari, M. Engholm, C. Beers, S. I. Nikolov, M. B. Stuart, E. V. Thomsen, J. A. Jensen, Curvilinear 3-D imaging using row-column addressed 2-D arrays with a diverging lens: Phantom study, IEEE Trans. Ultrason., Ferroelec., Freq. Contr. (2018) Submitted.

[6] S. Yang, W. Qin, H. Guo, T. Jin, N. Huang, M. He, L. Xi, Design and evaluation of a compound acoustic lens for photoacoustic computed tomography, Biomed. Opt. Express 8 (5) (2017) 2756-2765. doi:10.1364/BOE.8.002756.

[7] A. J. Devaney, H. Levine, Effective elastic parameters of random composites, Appl. Phys. Lett. 37 (4) (1980) 377-379. doi:10.1063/1.91949.

[8] T. N. Nguyen, M. Lethiecq, F. Levassort, L. Pourcelot, Experimental verification of the theory of elastic properties using scattering approximations in (0-3) connectivity composite materials, IEEE Trans. Ultrason., Ferroelec., Freq. Contr. 43 (4) (1996) 640-645.

[9] Y. Hosono, Y. Yamashita, K. Itsumi, Effects of fine metal oxide particle dopant on the acoustic properties of silicone rubber lens for medical array probe, IEEE Trans. Ultrason., Ferroelec., Freq. Contr. 54 (8) (2006) 1589-1595. doi:10.1109/TUFFC.2007.429.

[10] S. Rubinsztajn, L. S. Smith, C. E. Baumgartner, Silicone rubber compositions comprising bismuth oxide and articles made therefrom, uS Patent 8,389,627 (March 2013).

[11] H. Wang, W. Jiang, W. Cao, Characterization of lead zirconate titantae piezoceramic using high frequency ultrasonic spectroscopy, J. Applied Phys. 85 (12) (1999) 8083-8091. doi:10.1063/1.370646.

[12] D. T. Blackstock, Fundamentals of Physical Acoustics, John Wiley and Sons Inc., 2000, Ch. 3, pp. 111-112.

[13] M. Engholm, H. Bouzari, T. L. Christiansen, C. Beers, J. P. Bagge, L. N. Moesner, S. E. Diederichsen, M. B. Stuart, J. A. Jensen, E. V. Thomsen, Probe development of cmut and pzt row-column-addressed 2-d arrays, Sens. Actuators A: Phys. (2018) Submitted.

[14] J. A. Jensen, H. Holten-Lund, R. T. Nilsson, M. Hansen, U. D. Larsen, R. P. Domsten, B. G. Tomov, M. B. Stuart, S. I. Nikolov, M. J. Pihl, Y. Du, J. H. Rasmussen, M. F. Rasmussen, SARUS: A synthetic aperture realtime ultrasound system, IEEE Trans. Ultrason., Ferroelec., Freq. Contr. 60 (9) (2013) 1838-1852. 

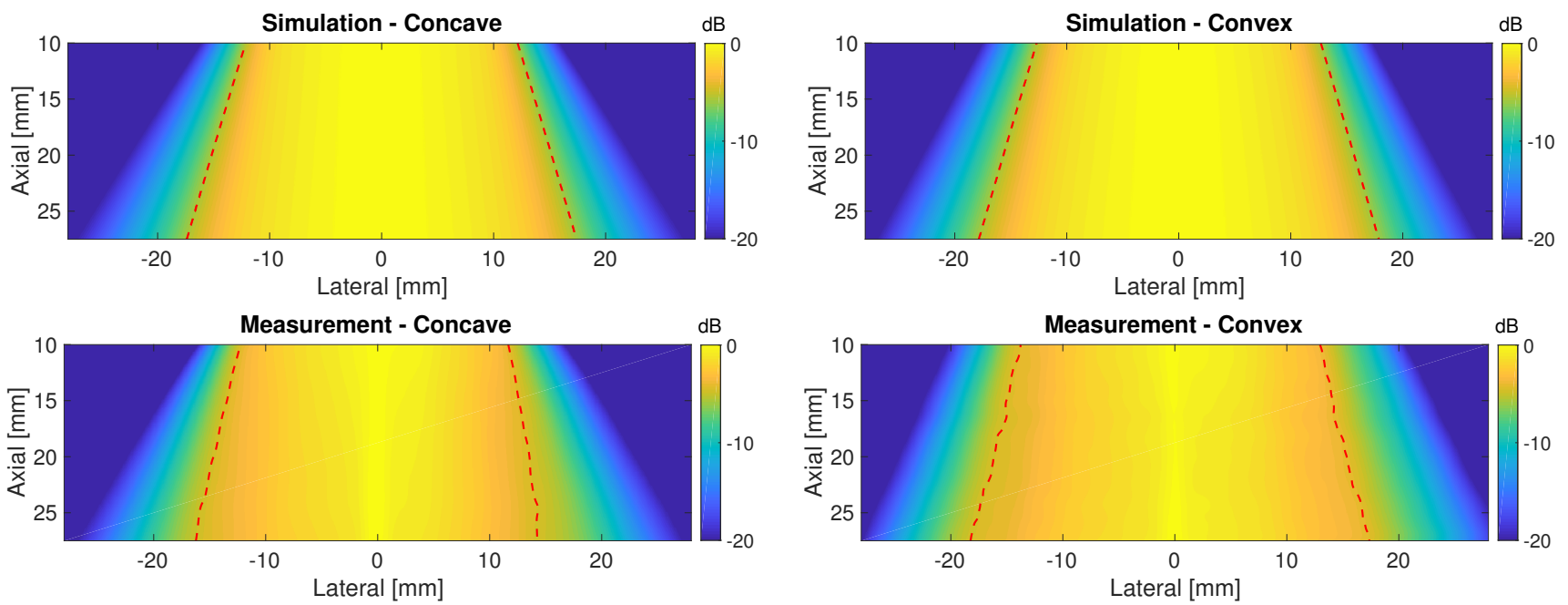

Figure 11: Beam profiles of measurements and simulations of the pressure amplitude recored at each position. The pressure is normalized to the maximum pressure amplitude at each depth, and the dashed red line shows the $-6 \mathrm{~dB}$ contour line, the FOV.

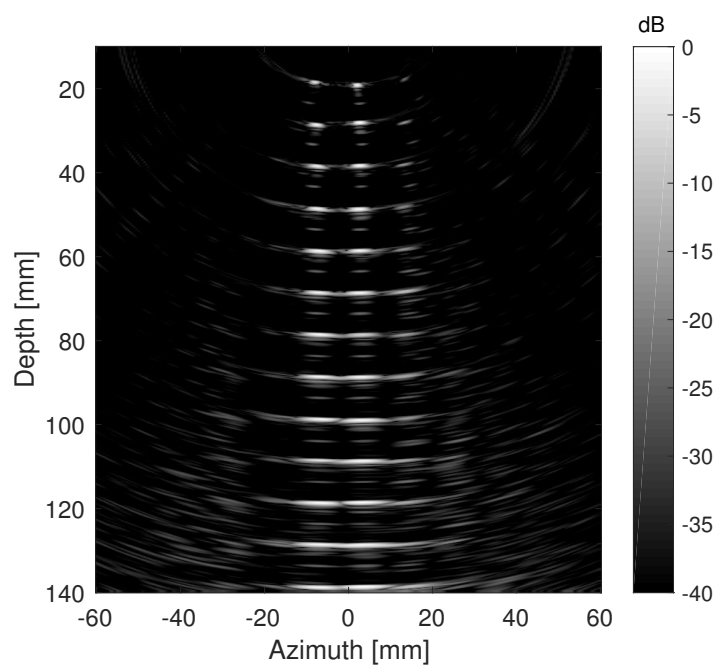

(a)

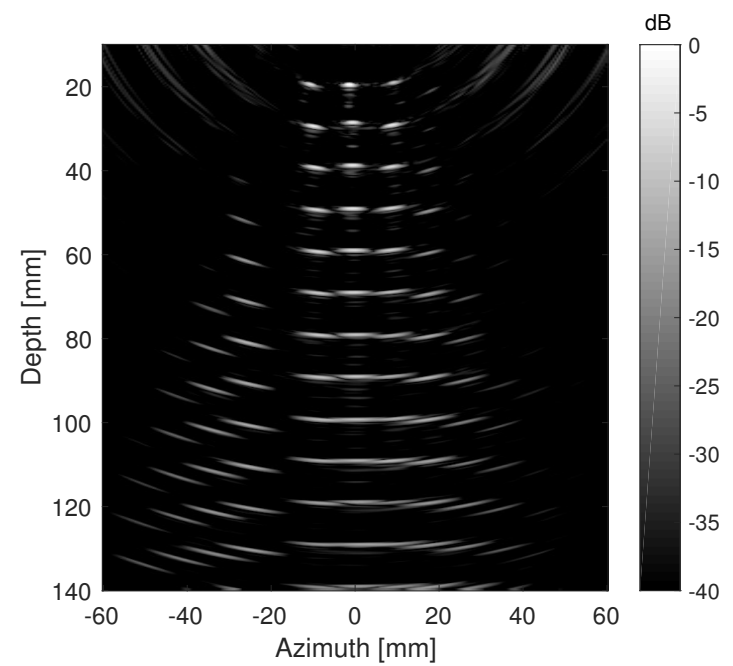

(b)

Figure 12: Beamformed image of a wire phantom. (a): Without the lens only the three center columns of the wire phantom are visible. (b): With the convex lens the wires within the FOV are all visible with an identical amplitude at the same depth. 
[15] D. G. Shombert, S. W. Smith, G. R. Harris, Angular response of miniature ultrasonic hydrophones, Med. Phys. 9 (4) (1982) 484-492. doi:10.1118/1.595114.

[16] Onda, Hydrophone handbook, Tech. rep., Onda Corporation, 1290 Hammerwood Ave, Sunnyvale, CA 94089 (2015). 